\title{
Role of adjunctive therapy with subantimicrobial doses of doxycycline in glycemic control (HbA1c) in patients with diabetes and endo-periodontal lesions to prevent sinus complications
}

\author{
ANAMARIA ZAHARESCU ${ }^{1 *}$, IOANA MÂRȚU ${ }^{2 *}$, ALEXANDRU-IONUȚ LUCHIAN $^{1 *}$, \\ MARIA-ALEXANDRA MÂRȚU ${ }^{1 *}$, IRINA-GEORGETA ȘUFARU ${ }^{1 *}$, CRISTIAN MÂRT,U $^{3 *}$ \\ and SORINA-MIHAELA SOLOMON ${ }^{1^{*}}$ \\ Departments of ${ }^{1}$ Periodontology, ${ }^{2}$ Implantology, ${ }^{3}$ ENT, 'Gr. T. Popa' \\ University of Medicine and Pharmacy, 700115 Iasi, Romania
}

Received September 25, 2020; Accepted October 27, 2020

DOI: $10.3892 / \mathrm{etm} .2021 .9708$

\begin{abstract}
The present study aimed to analyze local and regional changes in regards to odontogenic sinusitis in subjects with endo-periodontal lesions and diabetes mellitus and to investigate the effect on the level of glycemic control (glycated hemoglobin) that could be generated by adjunctive therapy with subantimicrobial doses of doxycycline. This study was performed on 51 subjects with diabetes who were divided into two therapeutic groups: 31 patients with diabetes (the study group) who underwent conventional endo-periodontal therapy and subantimicrobial doses of doxycycline and 20 patients with diabetes who followed only conventional endo-periodontal therapy (the control group). Patients underwent endodontic and periodontal clinical examination, with retro-dental-alveolar radiographs and $\mathrm{CBCT}$ examinations. For each patient, glycated hemoglobin A1c (HbAlc) was determined. This evaluation was performed at the beginning of the study, as well as 3,6 and 12 months after baseline. A significant percentage of patients, both in the study group and in the control group, showed radiological signs of odontogenic sinusitis, totaling 29 patients $(56.86 \%)$. Periodontal parameters revealed lower levels in patients who underwent the regimen which consisted of subantimicrobial doses of doxycycline; these results were maintained over the study period. Moreover, we demonstrated significantly reduced glycated hemoglobin levels throughout the study (12 months) in the doxycycline-treated group. This fact has far-reaching effects in the sphere of loco-regional
\end{abstract}

Correspondence to: Dr Cristian Mârțu, Department of ENT, 'Gr. T. Popa' University of Medicine and Pharmacy, 16 Universitatii Street, 700115 Iasi, Romania

E-mail: cristimartu@gmail.com

*Contributed equally

Key words: endo-periodontal lesions, diabetes mellitus, glycated hemoglobin, odontogenic sinusitis, subantimicrobial doses of doxycycline complications as well, and the risk of odontogenic sinusitis can be significantly reduced.

\section{Introduction}

The term endo-periodontal lesion appeared decades ago in order to describe a specific disease that affects the pulp and periodontal tissues simultaneously. Patients with diabetes are more prone to oral infections and periradicular lesions caused by changes in the immune system, qualitative and quantitative changes in the normal flora of the oral cavity and poor peripheral blood irrigation (1).

Furthermore, endo-periodontal lesions can be a risk factor for severe complications, such as odontogenic sinusitis. Inflammation and/or disruption of the Schneider membrane results in the inflammation of the mucosa and impaired mucociliary function in the maxillary sinus. Impaired mucociliary function results in altered mucus transport, impaired mucosal defense, blockage of sinus ostia and consecutive inflammatory processes (2).

Considering that diabetes adversely affects blood circulation or causes ischemia, sometimes necrotic tissue phenomena may occur (3). The possible connection between chronic oral inflammatory processes, such as apical periodontitis, endodontic status and systemic health, represents one of the most interesting aspects faced by the medical and dental scientific community; in order to achieve a proper healing potential, all parameters must be stabilized, in our case, inflammation and infection status in a diabetic field.

Glycemic control is essential to prevent diabetes-related morbidity and mortality. Increased glycated hemoglobin A1c (HbA1c) has been linked to diabetic microvascular and macrovascular complications and decreased HbA1c leads to reduced morbidity and mortality (4).

Doxycycline is an inexpensive, well-tolerated, broad-spectrum antibiotic that has the added benefit of being a potent inhibitor of host-derived matrix metalloproteinases (MMPs), even at subantimicrobial doses. Levels of MMP-class enzymes, including MMP-9 and MMP-8, have repeatedly been shown to be decreased in gingival tissues and periodontal 
lesions by subantimicrobial doses of doxycycline. Doxycycline in subantimicrobial doses (SDD) (Periostat ${ }^{\mathrm{TM}}$, CollaGenex Pharmaceuticals, Inc.; now Galderma R\&D) has been approved as an adjuvant to root planning and scaling for the treatment of periodontitis (5). The additional benefit of conventional subgingival debridement generated by doxycycline in subantimicrobial doses is due to the strong inhibition of extracellular matrix degradation, even in severe cases of periodontitis. The FDA (Food and Drug Administration) approved dose for subantimicrobial doxycycline is $20 \mathrm{mg}$ twice daily for up to 9 months. Antimicrobial action and side effects of antibiotics (for example, the emergence of antibiotic-resistant bacteria) do not occur at the recommended therapeutic doses (6).

Thus, the purpose of the study was to analyze local and regional changes (in terms of odontogenic sinusitis) in subjects with endo-periodontal lesions and diabetes mellitus and to investigate the effect on the level of glycemic control (glycated hemoglobin) that could be generated by adjunctive therapy with subantimicrobial doses of doxycycline.

\section{Patients and methods}

Patients. This study was performed on 51 subjects with diabetes mellitus type 2, divided into two therapeutic groups: 31 patients with diabetes (the SDD group) who underwent conventional endo-periodontal therapy and subantimicrobial doses of doxycycline and 20 patients with diabetes who followed only conventional endo-periodontal therapy (the control group). All of these patients had endo-periodontal lesions.

We excluded from the study patients with systemic diseases that are not a complication of diabetes mellitus, patients suffering from cancer, pregnant, breastfeeding or menopausal women, smokers, patients receiving dental treatment in the last 12 months or standard antibiotic regime in the last 2 months and those who had less than 20 remaining teeth.

In conducting the research, the ethical norms set out in the Declaration of Helsinki were respected. The present study was approved by the Ethics Committee of 'Grigore T. Popa' University of Medicine and Pharmacy (Iasi, Romania). Patients were informed about the aim of the study and each signed the informed consent required for study enrollment.

Clinical examination. Patients underwent a complex endodontic and periodontal clinical examination, which comprised vitality tests and the determination of the following periodontal parameters: Probing depth (PD), clinical periodontal attachment loss (CAL) and bleeding on probing (BOP).

The periodontal probing was performed with both the manual periodontal probe (CP-12, Hu-Friedy Mfg. Co., LLC) and an electronic one (Pa-On; Orange Dental GmbH \& Co., Germany). The probing depth, together with the loss of periodontal attachment, were measured in six points per tooth: Mesial-vestibular, central-vestibular, distal-vestibular, mesial-oral, central-oral, distal-oral. The BOP index was assessed by examination after $30 \mathrm{sec}$ of each probed site. Clinical examinations were conducted at baseline (T0), and at 3,6 and 12 months from baseline (T1,T2 and T3, respectively). All the data collected from the periodontal measurements are included in the patient's individual periogram.
Clinical examinations were supplemented with serial retro-dental-alveolar radiographs and CBCT examinations for the areas indicating radiological signs of odontogenic sinusitis.

Therapeutic procedure. All patients underwent non-surgical periodontal therapy, consisting of manual and ultrasonic scaling and root planning, with the help of curettes (Hu-Friedy Mfg. Co., LLC). For the mechanical instrumentation of root canals, the access cavity was made, the canals were permeabilized with Kerr needles (Kerr Corp., USA) no. 10 or 15. The instrumentation was performed with the manual ProTaper system (Dentsply Sirona), using the crown-down technique. Each patient was trained on the appropriate oral hygiene means.

These therapeutic procedures were performed for all subjects included in the study. In addition, patients in the SDD group underwent adjunct therapy to modulate the host's inflammatory response to bacterial aggression with subantimicrobial doses of doxycycline, $20 \mathrm{mg}$ twice daily, for 3 months. Adverse events were monitored and recorded throughout the study.

Each subject performed, at home, oral rinses with $0.10 \%$ chlorhexidine solution and $0.50 \%$ chlorobutanol $\left(\right.$ Eludril ${ }^{\circledR}$ ), twice/daily after dental brushing, for 2 weeks, starting on the first day of endo-periodontal treatment.

For patients with poor glycemic control, infection prophylaxis was also performed, with oral amoxicillin $2 \mathrm{~g}$, taken as a single dose, $1 \mathrm{~h}$ before each treatment session. The patients that required this type of prophylaxis treatment were excluded from the study.

Analysis of glycated hemoglobin. For each patient, glycated hemoglobin A1c (HbA1c) was determined. The method of determining HbAlc was immunoturbidimetric. This test does not interfere with other forms of pathological hemoglobin, such as carbamylated hemoglobin in uremic patients or acetylated hemoglobin caused by aspirin treatment; this is due to the high specificity of the anti-HbAlc antibodies for a 4 amino acid sequence at the $\mathrm{N}$-terminus of the $\beta$ chain in the glycated state. Therefore, this test determines 'real' HbAlc, as defined by the International Federation of Clinical Chemistry (IFCC) (7).

The quantification of glycated hemoglobin in total hemolyzed blood was based on a turbidimetric inhibition reaction. In a first step, glycated hemoglobin from the collected sample reacted with anti-HbA1c antibodies, with the formation of soluble antigen-antibody complexes. In the second step, polyhaptenes were added, which reacted with excess anti-HbAlc, by forming antibody-polyhaptene complexes, which were determined by immunoturbidimetry. The total hemoglobin concentration was determined in a separate channel. In the hemolyzed blood sample, the released hemoglobin was converted into a derivative, with a characteristic absorption spectrum; it was measured in two colors.

The percentage calculation of glycated hemoglobin was performed according to the Diabetes Controls and Complications Trial/National Glycohemoglobin Standardization Program (DCCT/NGSP) protocol (8), to which a correction formula was applied:

$\% \mathrm{HbA} 1 \mathrm{c}=(\mathrm{HbA} 1 \mathrm{c} / \mathrm{Hb}) \times 91.5+2.15$

This evaluation was performed at the beginning of the study and 3, 6 and 12 months after baseline. 
Table I. Demographic data of the study groups.

\begin{tabular}{|c|c|c|c|}
\hline Parameters & SDD group $(n=31)$ & Control group $(n=20)$ & Total $(n=51)$ \\
\hline Age (years) (mean \pm SD) & $52.17 \pm 9.72$ & $53.23 \pm 8.38$ & $52.97 \pm 10.21$ \\
\hline \multicolumn{4}{|l|}{ Sex, n $(\%)$} \\
\hline Male & $13(41.94 \%)$ & $8(40.00 \%)$ & $21(41.18 \%)$ \\
\hline Female & $18(58.06 \%)$ & $12(60.00 \%)$ & $30(58.82 \%)$ \\
\hline \multicolumn{4}{|l|}{ Provenance, $\mathrm{n}(\%)$} \\
\hline Urban & $21(67.74 \%)$ & $12(60.00 \%)$ & $33(64.71 \%)$ \\
\hline Rural & $10(32.26 \%)$ & $8(40.00 \%)$ & $18(35.29 \%)$ \\
\hline Odontogenic sinusitis prevalence, $\mathrm{n}(\%)$ & $18(58.06 \%)$ & $11(55.00 \%)$ & $29(56.86 \%)$ \\
\hline
\end{tabular}

SDD, subantimicrobial doses of doxycycline. No significant differences between groups at baseline were observed $(\mathrm{P}>0.05)$.

Statistical analysis. The data obtained during the course and at the end of the 12 months of the study were analyzed and statistically processed. The average values for the bleeding index, the probing depth and the level of clinical attachment loss per patient and at group and subgroup level were calculated. The Mann-Whitney test was used in order to detect significant differences between groups at different time points. The Wilcoxon test was used to evaluate changes over time. Values of $\mathrm{P}<0.025$ were considered statistically significant. The Mann-Whitney test with a significance level $\mathrm{P}<0.05$ was used to determine the significant differences between groups.

\section{Results}

The mean age of the 51 subjects was $52.97 \pm 10.21$ years. The group consisted of 30 female subjects $(58.82 \%)$ and 21 male subjects $(41.18 \%)$. Regarding the environment of origin, 33 subjects came from urban areas $(64.71 \%)$ and 18 from rural areas $(35.29 \%)$. Demographic data by study group are presented in Table I. Moreover, a significant percentage of patients, both in the study group and in the control group, showed radiological signs of odontogenic sinusitis, totaling 29 patients $(56.86 \%)$.

In terms of patient compliance, 34 subjects were initially included in the SDD group, but 3 of them $(8.82 \%)$ did not complete the regimen with subantimicrobial doses of doxycycline. All subjects included in the control group followed the study methodology.

Bleeding index. Following the evaluation of the bleeding index in the SDD group, we observed a significant decrease at the end of the therapy with subantimicrobial doses of doxycycline (T1), a decrease that continued at the 6 (T2) and 12 month (T3) assessments $(\mathrm{P}<0.001)$. For the control group, we noted significant decreases for the bleeding index after 3 months (T1); however, this followed an increasing trend at 6 (T2) and 12 months from baseline (T3), approaching the initial values (Fig. 1).

Probing depth. The determination of probing depth at 3 months from baseline (T1) revealed lower values in patients in the SDD group, even though it did not reach the statistical significance

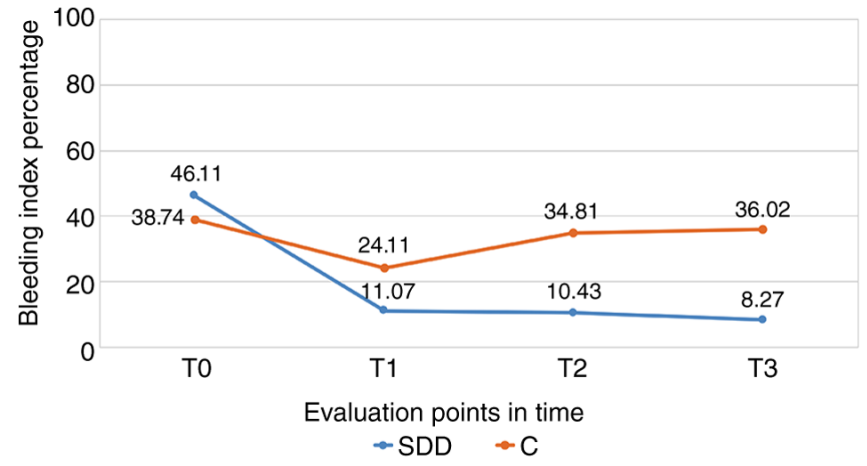

Figure 1. Bleeding index variation at evaluation time points: T0, baseline evaluation (before treatment); T1, evaluation after SDD (3 months from baseline); T2, evaluation at three months after SDD (6 months from baseline); T3, evaluation at nine months after SDD (12 months from baseline). SDD therapy exerted a significant decrease in bleeding, maintained through the study period (blue). In the control group (red), the bleeding index was lower immediately after standard therapy but there was a strong increase at 6 and 12 months from baseline, approaching the initial values. SDD, doxycycline at subantimicrobial doses.

threshold; these values continued to decrease throughout the study, the difference being significant at the 6 (T2) and 12 month (T3) assessments from baseline $(\mathrm{P}<0.05)$. Despite the average value of the probing depth being lower than the baseline at 3 months for the control group, this difference was not statistically significant. Moreover, the values increased at T2 and T3 evaluations (Fig. 2).

Loss of periodontal clinical attachment. When assessing the loss of periodontal clinical attachment after the completion of SDD therapy (T1), the value was lower, but did not reach a clinical significance threshold. Importantly, in the 6 (T2) and 12 month (T3) evaluations, we noted a decreased tendency of these values in the SDD group. In the control group, consisting of patients who only followed conventional therapy, CAL decreased significantly when assessed 3 months (T1) after the initial moment. Nevertheless, similarly to the other periodontal parameters, it showed an upward trend in the evaluations from 6 (T2) and 12 months (T3), the last of them revealing a value even higher than the initial one (Fig. 3). 


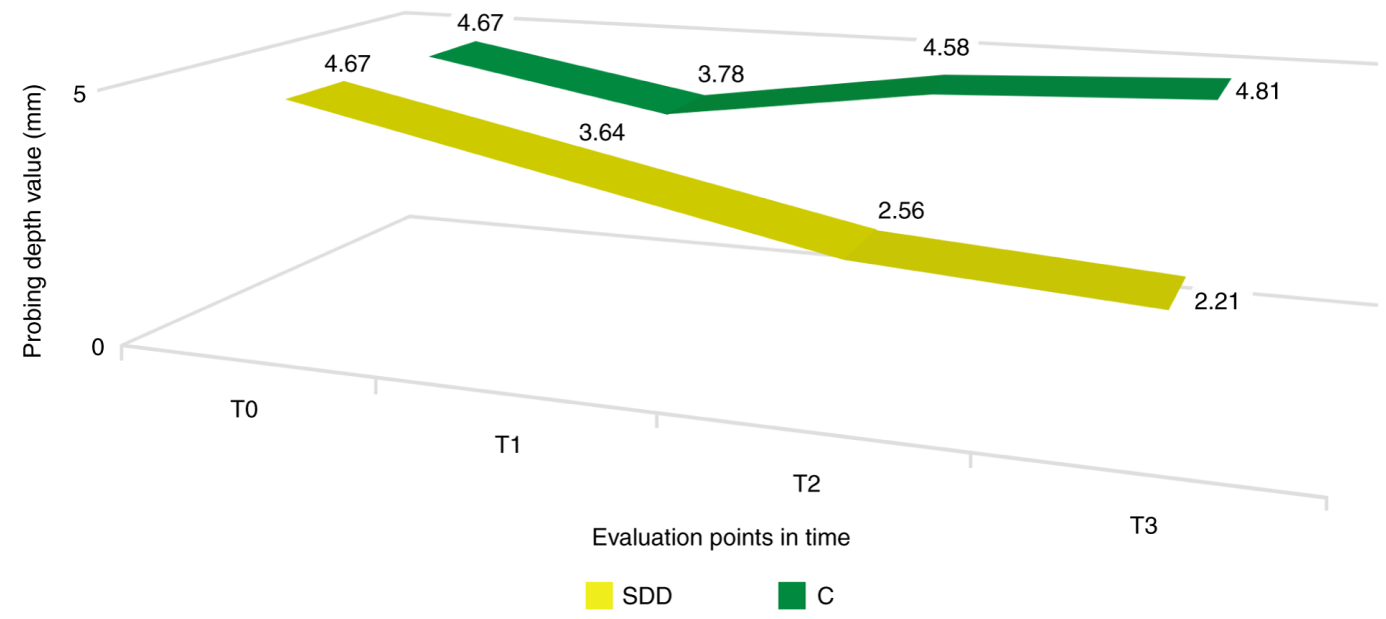

Figure 2. Probing depth variation at the evaluation time points: T0, baseline evaluation (before treatment); T1, evaluation after SDD ( 3 months from baseline); $\mathrm{T} 2$, evaluation at three months after SDD (6 months from baseline); T3, evaluation at nine months after SDD (12 months from baseline). In the SDD group the probing depth decreased throughout the study (yellow). In the control group the values increased at 6 and 12 months (green). SDD, doxycycline in subantimicrobial doses.

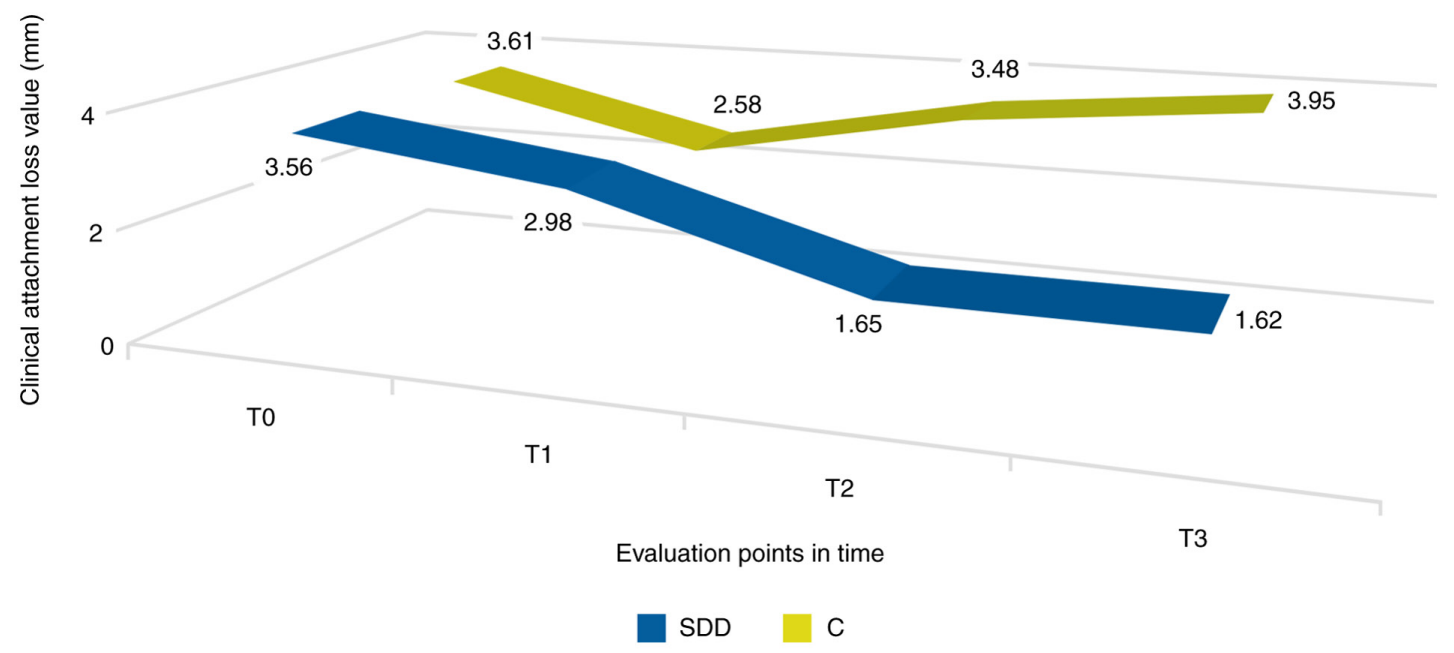

Figure 3. Clinical attachment loss (CAL) variation at evaluation time points: T0, baseline evaluation (before treatment); T1, evaluation after SDD (3 months from baseline); T2, evaluation at three months after SDD (6 months from baseline); T3, evaluation at nine months after SDD (12 months from baseline). In the SDD group in the 6 and 12 month evaluations we noted a deceasing tendency for the CAL values (blue). In the control group, CAL showed an upward trend in the evaluations at 6 and 12 months (yellow). SDD, doxycycline in subantimicrobial doses.

At the beginning, we did not note significant differences between groups for any of the analyzed periodontal parameters. At the end of the SDD therapy (T1), only the bleeding index showed significantly lower values for the SDD group compared to the control group ( $\mathrm{P}=0.0311)$, but 3 (T2) and 9 (T3) months after the completion of the SDD therapy, we observed significantly lower values for all the examined periodontal parameters.

Regarding the level of glycated hemoglobin, at T1we noted significant decreases for both study groups. The differences between the SDD group and the control group were significant when compared at the $\mathrm{T} 2$ and $\mathrm{T} 3$ assessments $(\mathrm{P}=0.0025$ and 0.0002 , respectively). For the group of patients with diabetes who underwent subantimicrobial doses of doxycycline therapy $\mathrm{HbAlc}$, it continued to decrease, while for the group of patients who only followed conventional therapy, these values began to increase, approaching the baseline values (Table II).

\section{Discussion}

Comparisons between patients with diabetes and those in the control group led to the observation that diabetes constitutes a risk factor for periodontal disease in general and for endo-periodontal lesions in particular. Oral disorders, such as periodontal disease, as well as diabetes, are multifactorial diseases (9). More obviously, diabetic patients are susceptible to various forms of periodontal disease, a particular importance being given to the diabetes-periodontal disease relationship, with the identification of patients who are more prone to these types of oral disorders (10).

Diabetes is known to decrease the host resistance to infections and diminish wound healing. Insulin is required for glucose uptake into cells and to provide an energy source for amino acid amelioration in protein synthesis, as well as for preventing lipolysis of adipose tissue. If insulin administration 
is not sufficient, then the basic cell functions of the body will be disrupted. Signs of host defense against microbes are well known: Impaired polymorphonuclear (PMN) leukocyte cell function with adhesion abnormalities, chemotaxis, phagocytosis, and intercellular destruction. Type $2 \mathrm{DM}$ is associated with a series of microvascular complications that most commonly affect the eyes and kidneys, and histopathological studies have shown internal ear nerve and vessels damage in subjects with diabetes (11).

An important complication related to the poor glycemic control, with great effect on the quality of life, bacterial, fungal or viral infections, are common in patients with diabetes and can affect the skin and soft tissue structure of the ear and nose. Both hypoglycemia and hyperglycemia have been associated with internal ear dysfunction and hearing can fluctuate with the level of glycemic control. The relationship between diabetes mellitus, sensory hearing loss and vestibular dysfunction is known, and histopathological changes of the temporal bone have been clearly documented (12).

The duration of diabetes is an important factor that causes the occurrence of microvascular complications of diabetes (13). It seems that the longer duration of diabetes mellitus predisposes to the development of deafness in many studies; however, a mild degree of hearing impairment has been detected in many children with diabetes lasting more than four years. Such an observation was unusual and may be explained by poor glycemic control. Elamin et al (14) confirmed the relationship of loss hearing in children and adolescents with type 1 diabetes mellitus at medium and high frequencies.

In diabetic patients with endo-periodontal lesions, periodontal therapy may have beneficial effects on glycemic control (10). This may be especially true for patients with relatively poorly controlled diabetes and more advanced periodontal destruction prior to treatment.

An understanding of the effects of other infections would be helpful in delimiting the mechanisms by which periodontal infection influences blood sugar. Acute bacterial and viral infections have been shown to increase insulin resistance and worsen metabolic control. This occurs in individuals with or without diabetes. Systemic infections increase tissue resistance to insulin, preventing the entry of glucose into the target cells, leading to an increase in blood sugar and requiring an increase in insulin production to maintain a normoglycemic state (10).

A systematic review examining the etiology of odontogenic sinusitis in a group of 674 patients showed that an iatrogenic etiology accounted for $65.7 \%$ of cases, apical periodontal pathology accounted for $25.1 \%$ of cases, and marginal periodontitis accounted for $8.3 \%$ of cases (15). This study further demonstrated that the most frequently affected maxillary teeth, in order of frequency, were the first molar $(35.6 \%)$, the second molar (22\%), the third molar (17.4\%) and the second premolar (14.4\%) (data not shown). Thus, there is an increased risk in patients with combined endo-periodontal lesions, especially if these lesions also affect the furcation area. In the present study, we noted a significant percentage of patients with endo-periodontal lesions who had radiological signs of odontogenic sinusitis, a diagnosis subsequently confirmed by CBCT examinations. In the context of the presence of diabetes, patients with endo-periodontal lesions are at high risk of local and loco-regional complications, including 
odontogenic sinusitis; this risk is amplified in cases of poor glycemic control. Therefore, modulation of the inflammatory response makes a significant contribution in mitigating these risks.

Several types of meta-analyses have confirmed that effective periodontal therapy may result in reduced glycated hemoglobin A1c (HbA1c). The first reported was performed on 10 interventional studies, with a combined population of 456 patients; the authors identified a weighted average HbAlc reduction of $0.66 \%$ as a result of periodontal therapy (although this failed to reach statistical significance) (16). In 2010, a meta-analysis of 5 studies involving 371 patients also reported a significant weighted average $\mathrm{HbA1c}$ reduction of $0.40 \%$ at a 3-9 months follow-up period (17).

The Cochrane collaboration reported studies that investigated the relationship between periodontal treatment and glycemic control in people with diabetes. Three studies were included in this meta-analysis that reported a significant reduction of $0.40 \% \mathrm{HbA1c}$ at 3-4 months after conventional periodontal therapy (18). The findings of these meta-analyses are supported by a population study of over 5,000 people with diabetes, reporting that patients who had at least one periodontal access surgery session had HbAlc levels that were $0.25 \%$ less than patients who had not undergone periodontal surgery (19).

Taken together, the evidence supports the idea that improvements in metabolic control can be anticipated following the effective treatment of periodontitis. The mechanisms by which this happens is not yet clear, but probably is due to reduced systemic inflammation (e.g., low serum concentrations of mediators such as TNF- $\alpha$ and IL-6), after treatment and resolution of periodontal inflammation (13). These observations are important as reductions in $\mathrm{HbA} 1 \mathrm{c}$ are associated with a reduced risk of diabetes complications. For example, it was found that each $1 \%$ reduction in $\mathrm{HbAlc}$ is associated with a $21 \%$ risk reduction for any diabetes-related complication, $21 \%$ for diabetes-related deaths, $14 \%$ for myocardial infarction, and $37 \%$ for microvascular complications (20).

Diabetes affects many functions of the immune system and is associated with delayed healing and compromised immune responses. Diabetes-induced changes in immune cell function produce an inflammatory immune cell phenotype (stimulation of pro-inflammatory cytokines from monocytes/polymorphonuclear leukocytes and inhibition of macrophage growth factors). This predisposes to chronic inflammation, progressive tissue breakdown and diminished tissue repair capacity (21).

Doxycycline and other tetracycline analogues have been shown to reduce tissue protein glycation in animals with streptozotocin-induced diabetes without apparent changes in serum glucose levels. Therefore, we hypothesized that doxycycline may be useful in the treatment of patients with diabetes by reducing protein glycation. The hypothesis of this study showed that SDD could play a role in reducing protein glycation in humans.

The implications of this study have far-reaching potential if the results are confirmed in larger and long-term studies. Firstly, SDD has already been approved for the adjuvant treatment of periodontitis. As patients with diabetes have a high risk of periodontitis, increased use of this type of therapy in the population will improve the results of periodontal treatment and may lead to improvements in diabetes outcomes. Secondly, we did not observe any increased incidence of adverse events in patients with type 2 diabetes who had SDD for three months (data not shown). Thirdly, subjects took stable doses of oral hypoglycemic agents and/or insulin and no adverse events were observed, indicating an apparent lack of adverse drug interactions between SDD and these agents.

As described in a larger number of studies on SDD in non-diabetic populations, no serious adverse events were observed in this study and SDD appeared to be well tolerated (22-24). Therefore, the use of SDD appears to be safe and effective for the treatment of endo-periodontal lesions in subjects with type 2 diabetes. However, these data should be interpreted with caution, given the small sample size. Clearly, larger studies are needed in subjects with type 2 diabetes to confirm whether this treatment is safe and effective for the treatment of endo-periodontal lesions in patients with type 2 diabetes and to test whether SDD is an effective adjuvant drug for the treatment of diabetes. It also remains to be determined whether long-term administration of SDD is safe and effective in reducing the complications of diabetes. However, based on these pilot data, longitudinal studies appear to be warranted.

Therefore, subantimicrobial doses of doxycycline generated favorable results for the evaluated periodontal parameters (bleeding index, probing depth and clinical periodontal attachment loss) and, unlike conventional therapy, these results were maintained over time.

Moreover, we demonstrated that adjunctive therapy with SDD had a clear contribution to improving glycemic control in patients with diabetes and endo-periodontal lesions, an improvement manifested by significantly reduced glycated hemoglobin levels throughout the study (12 months). This fact has far-reaching effects in the sphere of loco-regional complications as well and the risk of odontogenic sinusitis can be significantly reduced.

In addition, subantimicrobial dose therapy of doxycycline was well tolerated, with no adverse effects, which contributes to its recommendation in the therapeutic management of patients with diabetes mellitus and endo-periodontal lesions.

\section{Acknowledgements}

Professional editing, linguistic and technical assistance were performed by Irina Radu, Individual Service Provider.

\section{Funding}

The study was partially funded by a $\mathrm{PhD}$ scholarship provided to Ms. Anamaria Zaharescu by the 'Grigore T. Popa' University of Medicine and Pharmacy (Iasi, Romania).

\section{Availability of data and materials}

The data used and/or analyzed during the current study are available from the corresponding author on reasonable request.

\section{Authors' contributions}

AZ designed the experiments and performed the interventional study. IM contributed to the design of the study and 
data acquisition. AIL contributed to the data analysis and data interpretation, and edited the manuscript. MAM contributed to the data analysis and to the design of the study. IGS contributed to the design of the study and edited the manuscript. $\mathrm{CM}$ acquired and analyzed the data, and wrote the main manuscript text. SMS designed the study, interpreted the data and provided archive data. All authors read and approved the manuscript and agree to be accountable for all aspects of the research in ensuring that the accuracy or integrity of any part of the work are appropriately investigated and resolved.

\section{Ethical approval and consent to participate}

The present study was approved by the Ethics Committee of 'Grigore T. Popa' University of Medicine and Pharmacy (Iasi, Romania). All protocols were in accordance with the provisions of the Declaration of Helsinki. The purpose and safety aspects of the study were explained to all patients and written informed consent was included in the documents of each patient.

\section{Patient consent for publication}

Not applicable.

\section{Competing interests}

The authors declare that they have no competing interests.

\section{References}

1. Al-Fouzan KS: A new classification of endodontic periodontal lesions. Int J Dent 2014: 919173, 2014.

2. Gamba P: Odontogenic maxillary cysts post-dental implant: Proposal of new radiological/clinical classification. Int J Innovative Res Med Sci 10: 431-438, 2016.

3. Dhoum S, Laslami K, Rouggani F, El Ouazzani A and Jabri M: Endo-Perio lesion and uncontrolled diabetes. Case Rep Dent 2018: 7478236, 2018.

4. Khaw KT, Wareham N, Luben R, Bingham S, Oakes S, Welch A and Day N: Glycated haemoglobin, diabetes, and mortality in men in Norfolk cohort of European Prospective Investigation of Cancer and Nutrition (EPIC-Norfolk). BMJ 322: 15-18, 2001

5. Caton J and Ryan ME: Clinical studies on the management of periodontal diseases utilizing subantimicrobial dose doxycycline (SDD). Pharmacol Res 63: 114-120, 2011.

6. Engebretson SP and Hey-Hadavi J: Sub-antimicrobial doxycycline for periodontitis reduces hemoglobin A1c in subjects with type 2 diabetes: A pilot study. Pharmacol Res 64: 624-629, 2011.

7. Groche D, Hoeno W, Hoss G, Vogt B, Herrmann Z and Witzigmann A: Standardization of two immunological HbAlc routine assays according to the new IFCC reference method. Clin Lab 49: 657-661, 2003.

8. Little R, Rohlfing C, Wiedmeyer H, Myers G, Sacks D and Goldstein D: The National Glycohemoglobin standardization program: A five-year progress report. Clin Chem 47: 1985-1992, 2001.
9. Liccardo D, Cannavo A, Spagnulo G, Ferrara N, Cittadini A, Rengo C and Rengo G: Periodontal disease: A risk factor for diabetes and cardiovascular disease. Int J Mol Sci 20: 1414, 2019.

10. Mealey BL and Oates TW: Diabetes mellitus and periodontal diseases. J Periodontol 77: 1289-1303, 2006.

11. Graves DT, Ding Z and Yang Y: The impact of diabetes on periodontal disease. Periodontol 82: 214-224, 2020.

12. Gazzaz ZJ, Makhdom MN, Dhafar KO, Maimini O, Farooq MU and Rasheed A: Patterns of otorhinolaryngological disorders in patients with diabetes. Int Med J Malaysia 10: 13-16, 2011.

13. American diabetes association: Diagnosis and classification of diabetes mellitus. Diabetes Care 37: 581-590, 2014.

14. Elamin A, Fadlallah M and Tuvemo T: Hearing loss in children with type 1 diabetes. Indian Pediatr 42: 15-21, 2005.

15. Lechien JR, Filleul O, de Araujo PC, Hsieh JW, Chantrain G and Saussez S: Chronic maxillary rhinosinusitis of dental origin: A systematic review of 674 patient cases. Int J Otolaryng 2014: 465173, 2014.

16. Janket SJ, Wightman A, Baird AE, Van Dyke TE and Jones JA: Does periodontal treatment improve glycemic control in diabetic patients? A meta-analysis of intervention studies. J Dent Res 84: 1154-1159, 2005

17. Teeuw WJ, Gerdes VEA and Loos BG: Effect of periodontal treatment on glycemic control of diabetic patients: A systematic review and meta-analysis. Diabetes Care 33: 421-427, 2010.

18. Simpson TC, Needleman I, Wild SH, Moles DR and Mills EJ: Treatment of periodontal disease for glycaemic control in people with diabetes. Cochrane Database Syst Rev 5: CD004714, 2010.

19. Spangler L, Reid RJ, Inge R, Newton KM, Hujoel P, Chaudhari M, Genco RJ and Barlow WE: Cross-sectional study of periodontal care and glycosylated hemoglobin in an insured population. Diabetes Care 33: 1753-1758, 2010.

20. Stratton IM, Adler AI, Neil HA, Matthews DR, Manley SE, Cull CA, Hadden D, Turner RC and Holman RR: Association of glycaemia with macrovascular and microvascular complications of type 2 diabetes (UKPDS 35): Prospective observational study. BMJ 321: 405-412, 2000.

21. Nayak AU, Nevill AM, Bassett P and Singh BM: Association of glycation gap with mortality and vascular complications in diabetes. Diabetes Care 36: 3247-3253, 2013.

22. Caton JC, Ciancio SG, Blieden TM, Bradshaw M, Crout RJ, Hefti AF, Massaro JM, Polson AM, Thomas J and Walker C: Treatment with subantimicrobial dose doxycycline improves the efficacy of scaling and root planing in patients with adult periodontitis. J Periodontol 71: 521-532, 2000.

23. Walker C, Puumala S, Golub LM, Stoner JA, Reinhardt RA, Lee $\mathrm{H}$ and Payne JB: Subantimicrobial dose doxycycline effects on osteopenic bone loss: Microbiologic results. J Periodontol 78: 1590-1601, 2007.

24. Payne JB, Golub LM, Stoner JA, Lee H, Reinhardt RA, Sorsa T and Slepian MJ: The effect of subantimicrobial-dose-doxycycline periodontal therapy on serum biomarkers of systemic inflammation: A randomized, double-masked, placebo-controlled clinical trial. J Am Dent Assoc 142: 262-273, 2011.

This work is licensed under a Creative Commons Attribution-NonCommercial-NoDerivatives 4.0 International (CC BY-NC-ND 4.0) License. 